DYNAMICAL INTERPRETATIONS OF THE GALACTIC CENTER REGION

Robert H. Sanders

Kapteyn Astronomical Institute, Groningen

I will define the central region of the Galaxy as being the inner four kiloparsecs. The distinguishing characteristics of this region are:

1) The dominance of a central spheroidal component in the mass distribution -- a bulge.

2) An apparent deficiency of gas, at least between radii of $500 \mathrm{pc}$ and $4000 \mathrm{pc}$.

3) High non-circular gas velocities.

Now let us consider these characteristics in some detail.

\title{
1. THE BULGE
}

The rotation curve, as observed in the $21-\mathrm{cm}$ line of neutral hydrogen, gives some indication of the form of the gravitational field and, hence, the mass distribution in this region. Figure 1 is the rotation curve in the inner few kiloparsecs (solid line). Inside $1 \mathrm{kpc}$ the curve is essentially that of Rougoor and Oort (1960); and beyond $1 \mathrm{kpc}$ the curve is a fit to the data presented by Simonson and Mader (1973). The existence of an inner centrally condensed component in the mass distribution - a component which is distinct from the more extended disk - is implied by the presence of the inner peak in the rotation curve; specifically, the rise to $260 \mathrm{~km} \mathrm{~s}^{-1}$ at $800 \mathrm{pc}$.

Independent confirmation of the centrally condensed component came in 1968 with the near infrared observations of Becklin and Neugebauer. They discovered a source of extended emission at $2.2 \mu$ which they interpreted as being starlight and, therefore, an indication of the density distribution of stars within the galactic nuclear region. liaking a few reasonable assumptions one can easily convert this $2.2 \mu$ intensity distribution into a stellar density distribution. This has been done by Becklin and Neugebauer (1968), Oort (1971), and Sanders and Lowinger (1972). In particular, Sanders and Lowinger derive a stellar density distribution in the inner 50 pc described by the formula

$$
\rho=\frac{7.6 \times 10^{5}}{\mathrm{r}^{1.8}} \mathrm{M}_{\odot} \mathrm{pc}^{-3}
$$

383

W. B. Burton (ed.), The Large-Scale Characteristics of the Galaxy, $383 \cdots 392$.

Copyright $\odot 1979$ by the IAU. 


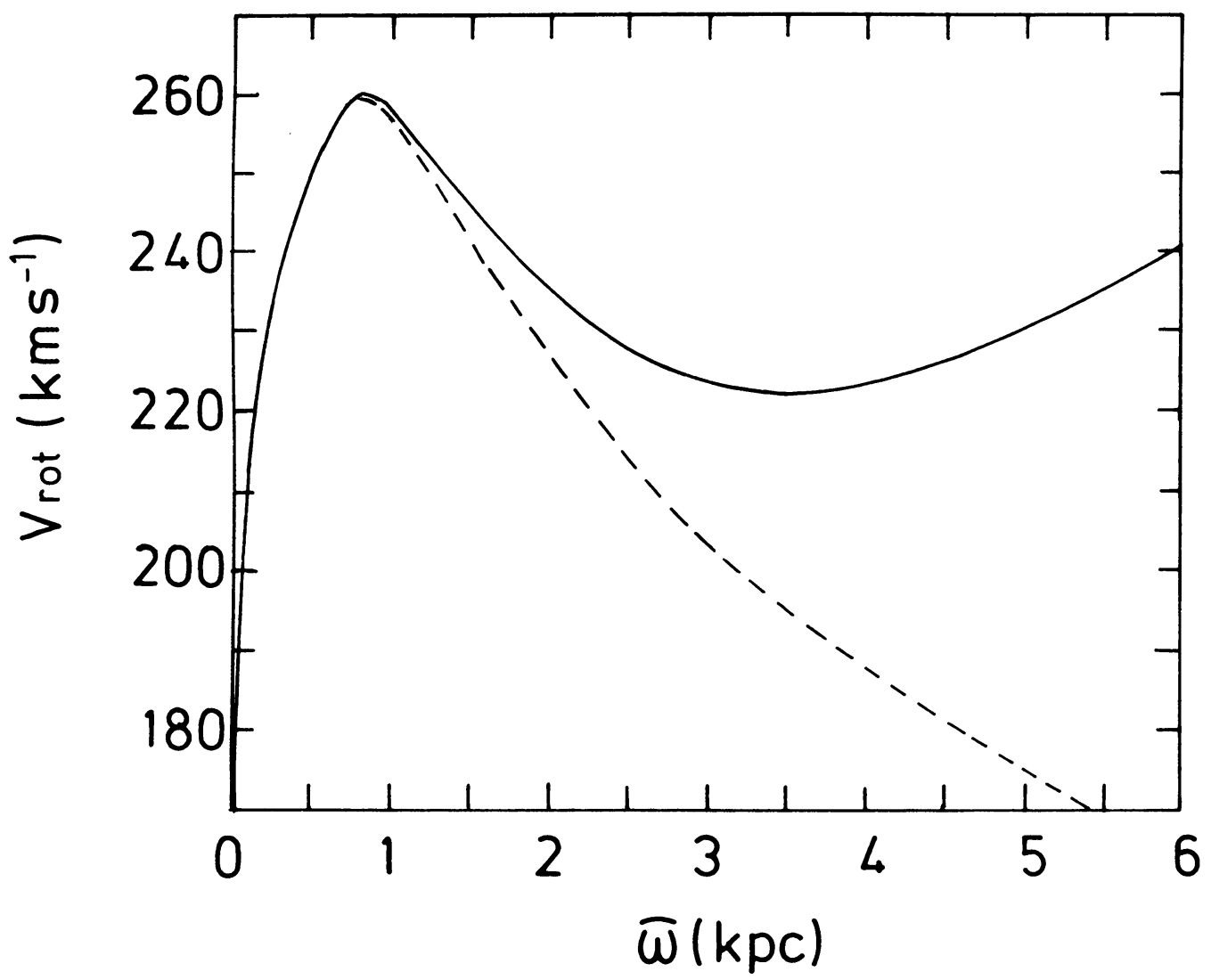

Figure 1. Rotation curve (solid line) in the inner region of the Galaxy and rotation curve (dashed line) of bulge-halo component.

If we extrapolate this density law out to $800 \mathrm{pc}$ (an extrapolation which seems justified by comparison with M31), we may derive a rotation curve which is practically indentical to the observed $21-\mathrm{cm}$ curve at radii less than $800 \mathrm{pc}$. Thus, there appears to be agreement between two independent observational determinations of the mass distribution in the bulge. This gives us some confidence in both techniques: that the near infrared really does reveal the distribution of old stars near the center and that the neutral hydrogen velocity field inside $800 \mathrm{pc}$ is dominated by circular motion and truly reflects the gravitational field; in other words, that the HI motions are not drastically affected by some presumed general expansion motion inside $300 \mathrm{pc}$.

Now I would like to emphasize two important aspects of this observed central spheroid:

a) This spheroid, or bulge, could very likely be the central core 
of an extended spheroidal galactic halo; a halo with a mass sufficient to stabilize the disk. The density law derived from the Becklin-Neugebauer observations (Equation 1) is basically that of an isothermal sphere. But if the bulge is to continue beyond $1000 \mathrm{pc}$, it must have a steeper density law in order to be consistent with the observed rotation curve; specifically with the dip in the rotation curve between one and six kpc (Fig. 1). The likely tracers of the halo, the low-metal RR Lyrae stars and the globular clusters, would suggest an $\mathrm{r}^{-3}$ density law beyond one $\mathrm{kpc}$ (Oort 1965, Oort and Plaut 1975). Fitting an $\mathrm{r}^{-3}$ law smoothly to the bulge density at $800 \mathrm{pc}$ (that is, the density derived from extrapolation of the near infrared observations), we find

$$
\rho=\frac{10^{9}}{\mathrm{r}^{3}} \mathrm{M}_{\odot} \mathrm{pc}^{-3}
$$

for $\mathrm{r}>800 \mathrm{pc}$.

The dashed 1ine in Figure 1 is the rotation curve for this bulgehalo component. The difference between the bulge-halo rotation curve and the observed rotation curve is presumably due to an extended disk component, but the significant point is that we can smoothly tie an extended halo onto the bulge without violating the observed rotation law. Moreover, in the context of this model, the mass of the bulge-halo inside $10 \mathrm{kpc}$ is comparable to the mass of the disk, both being about $5 \times 10^{10} \mathrm{M}_{\odot}$. Therefore, the bulge-halo has a mass which is sufficient to stabilize the disk against the violent bar-forming modes discussed by Ostriker and Peebles (1973).

b) The bulge-halo is probably axisymmetric and possibly nearly spherically symmetric. The halo tracers, specifically the low-metal RR Lyrae stars from $1 \mathrm{kpc}$ to $5 \mathrm{kpc}$, seem to have a spherically symmetric distribution (Oort and Plaut 1975). In M31 the visual isophotes become almost circular within a few hundred parsecs of the center where the bulge dominates the light distribution (Light, Danielson, and Schwarzschild 1975). This supports the hypothesis that the bulges of spiral galaxies are, in general, hot axially symmetric systems.

The probable axial symmetry of the bulge in our Galaxy has implications regarding the interpretation of non-circular gas velocities in the central region. It has been suggested that such high non-circular velocities may be due to the action of a rotating oval or bar-like distortion. If so, then from arguments given above, it is probably the disk component and not the bulge-halo which is ovally distorted. But the disk makes a significant contribution to the gravitational field only at radii greater than about $3 \mathrm{kpc}$. Therefore, it is possible that an oval distortion of the disk could drive non-circular motions associated with the $3 \mathrm{kpc}$ arm, but an oval distortion is not likely to be responsible for the high non-circular velocities observed within 200 pc of the center.

\section{THE OVERALL GAS DISTRIBUTION}

The second general characteristic of the galactic center region is the deficiency of gas inside four kiloparsecs. Figure 2 shows the overall radial distribution of gas in the Galaxy (Gordon and Burton 1976). The 


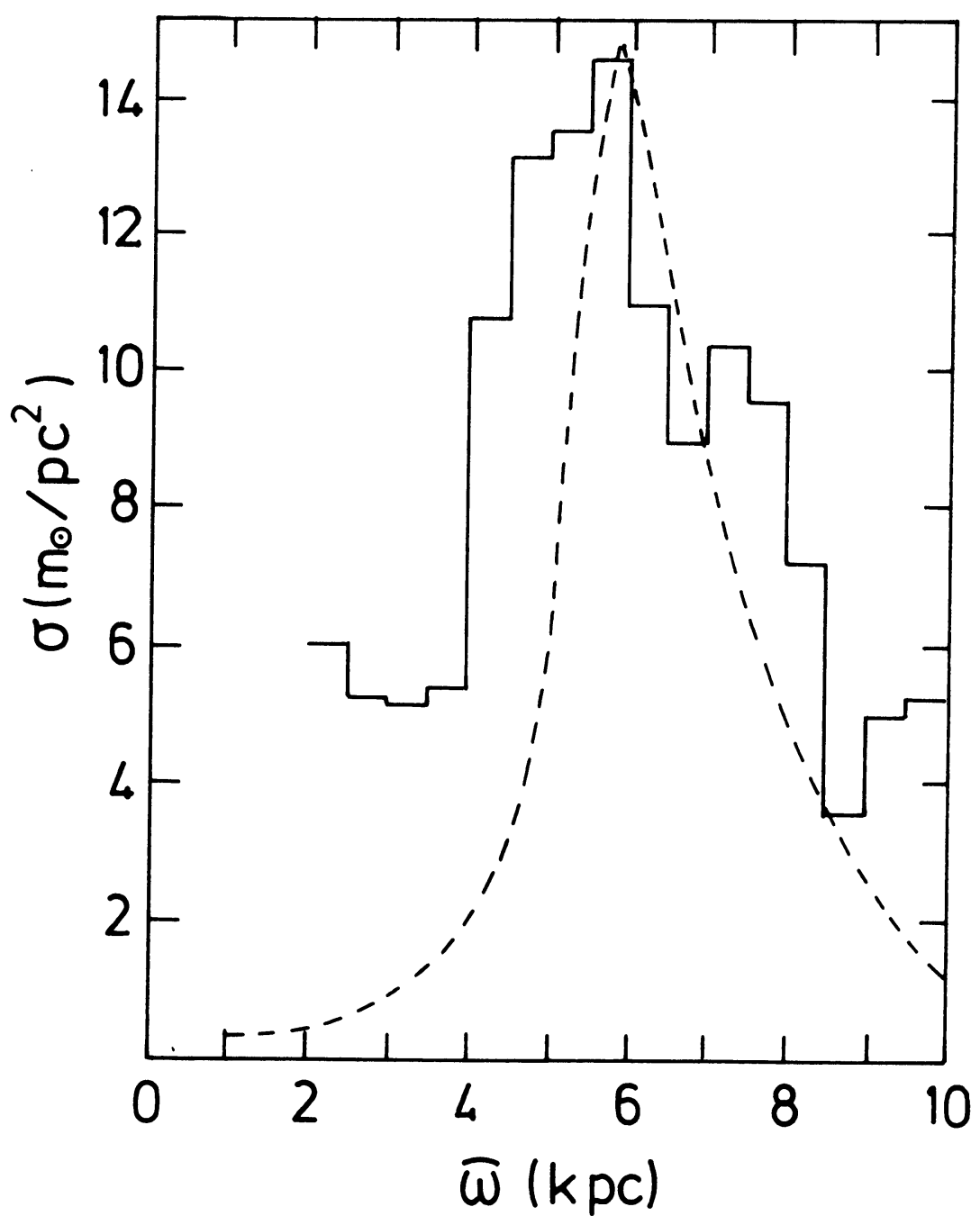

Figure 2. Radial distribution of gas surface density (Gordon and Burton 1976). Dashed line is distribution resulting from turbulent transfer of angular momentum.

gas surface density increases inward to about $5 \mathrm{kpc}$ and then decreases rather abruptly inside $4 \mathrm{kpc}$; i.e., there is a hole in the total gas density distribution. In fact, there is quite a bit of gas in the central regions but it is all in the form of massive molecular clouds within a few hundred parsecs of the center. Bania (1977) estimates that there may be $7 \times 10^{8} \mathrm{M}_{\odot}$ of molecular gas within $500 \mathrm{pc}$ of the center. If this gas were distributed evenly throughout the inner $4 \mathrm{kpc}$, it would in some sense fill up the hole. This suggests that inside $4 \mathrm{kpc}$, the gas has 
experienced some efficient loss or transfer of angular momentum.

One possible mechanism is an effective breaking of the gas due to interaction with a bar (Matsuda and Nelson 1977). This suggestion is based upon numerical calculations which contain a large artificial viscosity; therefore, it is very likely that this effect has been overestimated.

A second possibility is the outward transfer of angular momentum by a turbulent shear viscosity which is due to large-scale cloud-cloud interactions (Lynden-Bell 1969, Icke 1978). Given such a viscosity, there are two equations for the time development of the gas surface density, the equations of motion and continuity. A numerical solution of these two equations, appropriate for the Galactic rotation curve (or shear), is shown by the dashed 1 ine in Figure 2. The initial gas surface density distribution is assumed to be constant, and the distribution shown here develops over a period of $10^{10}$ years (assuming a constant cloud mean-freepath of $200 \mathrm{pc}$ and a random velocity of $10 \mathrm{~km} \mathrm{~s}^{-1}$ ). The general trends in the radial distribution of gas are accounted for in such a picture. In particular there is an accumulation of gas near $5 \mathrm{kpc}$ and severe deficiency in the inner regions. The gas which was originally present inside $4 \mathrm{kpc}$ has been transferred inward, through the inner boundary in this calculation (at $\mathrm{r}=1 \mathrm{kpc}$ ).

It should be emphasized that for a simple single-peaked rotation curve, the shear, and, as a consequence, the rate of transfer of the angular momentum approaches zero. Therefore, if the galactic rotation curve had only one outer peak, the inner regions would not be depleted of gas. In order to deplete the inner $4 \mathrm{kpc}$ by inflow, the galaxy must have a central peak in the rotation curve or, in other words, a massive central spheroid or bulge. It is the effect of the bulge on the rotation curve that creates strong shear in the inner regions. This would seem to be consistent with the observations of Bosma (1978) who has pointed out that central holes in the neutral hydrogen distribution tend to be present in galaxies with conspicuous bulges.

\section{NON-CIRCULAR GAS VELOCITIES}

Perhaps the most interesting characteristic of the galactic center region is the presence of high peculiar or non-circular gas velocities. (The relevant observations have been extensively reviewed by Oort, 1977.) For simplicity, I would like to divide the galactic center region into two sub-regions. Such a division is necessary because I will propose that the likely mechanism for excitation of high non-circular velocities is different in these two regions. Region $I$ is between radii of $3 \mathrm{kpc}$ and $4 \mathrm{kpc}$ and region II is within $200 \mathrm{pc}$ of the center.

a) Region I: This is the region of the $3-\mathrm{kpc}$ arm, a feature seen in neutral hydrogen and molecular line observations over at least $20^{\circ}$ of galactic longitude. At zero longitude the arm has a velocity of $53 \mathrm{~km} \mathrm{~s}^{-1}$ directed radially outward from the center. Now let us consider two suggested mechanisms for the origin of the large peculiar velocity of this feature: explosions and bars.

It is not likely that the 3-kpc arm is caused by expulsions or super explosions at the galactic center as suggested in the work of Van der Kruit 
(1971) or Sanders and Prendergast (1974). The energies required to excite radial gas motions so far from the center are enormous -- in excess of $10^{58}$ ergs for a symmetrical event. More significantly, vast amounts of gas must be ejected, -- $10^{8} \mathrm{M}_{\odot}$ per event. Given 100 such events over the lifetime of the galaxy (such a frequency would be necessary to maintain the observed non-circular motion for a reasonable fraction of the time), the total mass ejected from the central region would be on the order of $10^{10} \mathrm{M}_{\odot}$. Viscous transfer of angular momentum, the process described above, would fail by a factor of 100 to supply this amount of gas. Apart from these considerations, there is no independent evidence that events of this magnitude occur in the central regions of our own Galaxy.

The most likely explanation of the $3-\mathrm{kpc}$ arm phenomenon is gas flow on elliptical stream lines maintained by a bar or oval distortion of the disk component of the galaxy. This was a suggestion first made by Kerr (1968) and later modelled kinematically by Peters (1975) on the basis of a flow pattern suggested by Roberts (1971). I have recently done timedependent gas dynamical calculations of flow in the central region of a galactic gravitational field which is ovally distorted. The form of the axisymmetric potential was taken to mock up the central region of the galaxy; i.e., there are two components, bulge-halo and disk, with a rotation curve identical to that shown in Figure 1. The disk component was given a slight oval distortion by adding $\cos 2 \theta$ variation to the potential. Only.the disk was distorted, -- not the bulge. The angular velocity of the distortion is $15 \mathrm{~km} \mathrm{~s}^{-1} \mathrm{kpc}^{-1}$; hence, an inner resonance occurs between $3 \mathrm{kpc}$ and $4 \mathrm{kpc}$. The strength of the perturbation was chosen such that the $\theta$ force at $4 \mathrm{kpc}$ is about $10 \%$ of the mean axisymmetric force at that radius. Within $2 \mathrm{kpc}$ of the center the $\Theta$ force is less than $2 \%$ of the axisymmetric force due to the dominance of the axisymmetric bulge in this region. Gas motions are numerically followed until a quasi-steady state is reached in the rotating frame of the distortion. The steady state density distribution is that of an open trailing spiral. The steady state gas flow is illustrated in Figure 3. This is a contour map of the radial (or non-circular) component of the gas velocity. Solid lines are outflow, dashed lines are inflow. We see the "quadrupolar" pattern of non-circular velocities which is characteristic of flow on highly elliptical streamlines. In the inner 1000 pc (where the bulge dominates) the motion is predominately circular. The significant point is that a rather weak oval distortion can produce non-circular velocities of the observed magnitude. Moreover, if we view this flow pattern along the indicated line-of-sight (dotted 1 ine), we reproduce $21-\mathrm{cm}$ profiles of the 3-kpc arm component along its observed range of longitude. One problem with this model, of course, is that we would also predict an equal positive velocity counterpart to the $3-\mathrm{kpc}$ arm. This is a difficulty of any model with $180^{\circ}$ symmetry and may imply the existence of large-scale systematic asymmetries in the gravitational field near the center.

b) Region II: This is the region of the massive molecular clouds within $200 \mathrm{kpc}$ of the center. Here we find the great Sgr A and Sgr B2 complexes as well as the "expanding ring" first noticed by Scoville (1972) and Kaifu et al. (1972). This latter feature has been fit by a kinematic model consisting of an expanding, rotating ring. The ring has a radius of 


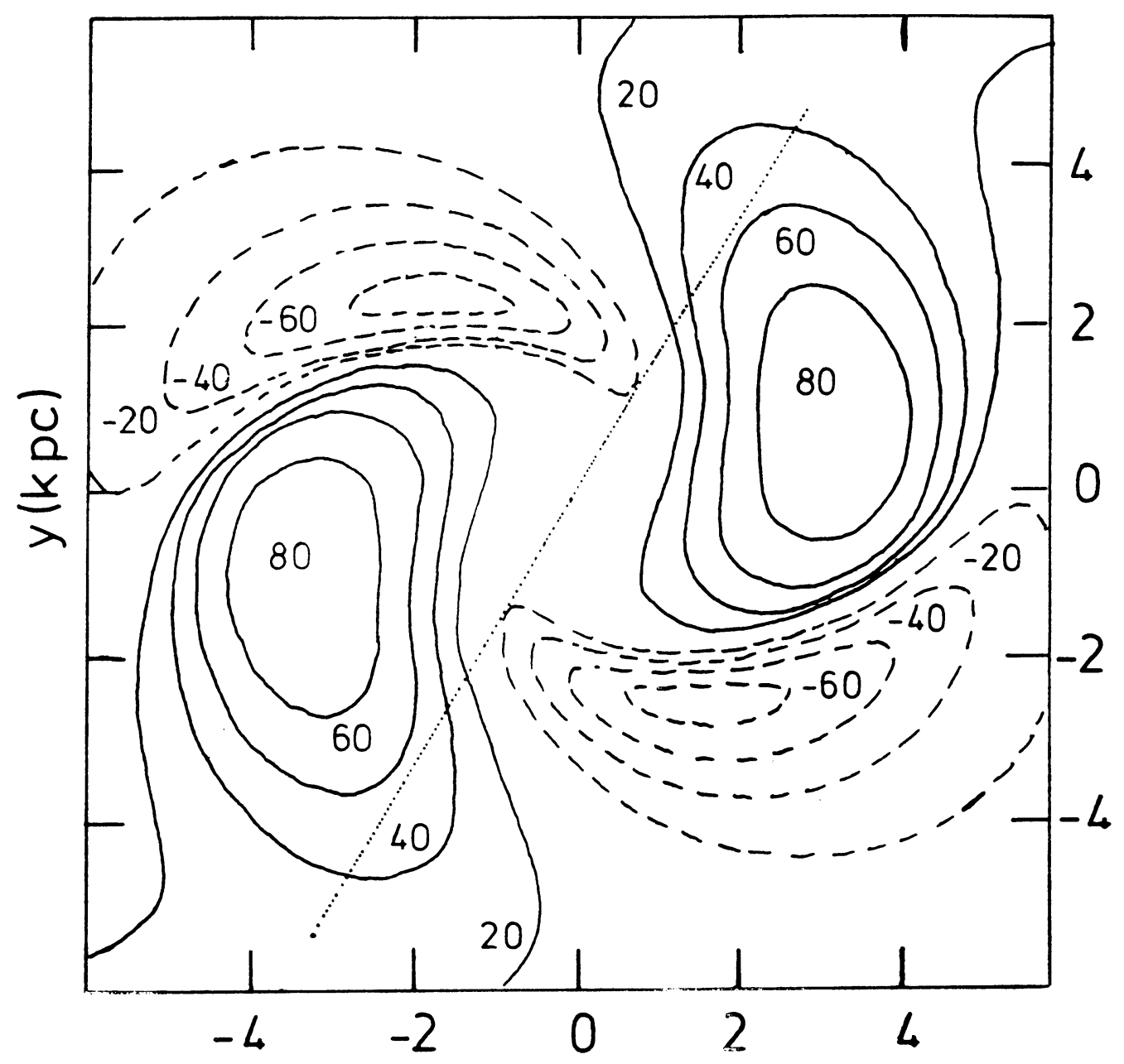

Figure 3. Contour map of non-circular velocity resulting from a rotating oval distortion in the inner $6 \mathrm{kpc}$ of the Galaxy. Velocities are in $\mathrm{km} \mathrm{s}^{-1}$.

$190 \mathrm{pc}$, an expansion velocity of $150 \mathrm{~km} \mathrm{~s}^{-1}$, and a rotational velocity of $65 \mathrm{~km} \mathrm{~s}^{-1}$. Its mass is on the order of $10^{7} \mathrm{M}$ (Bania 1977) implying a kinetic energy in expansion motion of $10^{54}$ ergs.

These extremely high systematic non-circular velocities could result either from the effects of a bar or from an explosive process. As discussed above, the overwhelming dominance of the axisymmetric bulge component in this region would seem to rule out a direct gravitational mechanism, such as a rotating bar distortion. Moreover, for a bar to have a strong resonance effect in this region, it would have to be rotating very rapidly, with an angular velocity well in excess of $100 \mathrm{~km} \mathrm{~s}^{-1}$. Therefore, such an inner bar would be dynamically distinct either from 
the bar presumably responsible for the $3 \mathrm{kpc}$ arm or from the outer spiral structure. While a little, fast bar in the inner $200 \mathrm{pc}$ cannot be ruled out, the dominance of the hot axisymmetric bulge favors, in my opinion, an explosion hypothesis for the non-circular gas motions in this region. Above, we have discussed a mechanism by which more than $10^{8} \mathrm{M}_{\odot}$ could accumulate near the center over a galactic time scale. Such an accumulation could provide fuel for a variety of explosive mechanisms. To maintain the observed non-circular motions inside $200 \mathrm{pc}$, the explosive "events" would have to recur on time scales of $10^{7}$ years and provide at least $10^{55}$ ergs. Since our galactic nucleus is not spectacularly luminous at the present time $\left(<10^{40}\right.$ ergs $\left.\mathrm{s}^{-1}\right)$, the radiation associated with such an event should be quite short lived ( $\leqslant 10^{5}$ years), and what we presently observe is the hydrodynamic relic or fossil of the most recent period of activity.

\section{REFERENCES}

Bania, T.M.: 1977, Astrophys.J. 216, 381 .

Becklin, E.E., Neugebauer, G.: 1968, Astrophys.J. 151, 145.

Bosma, A.: 1978, Dissertation, University of Groningen.

Gordon, M.A., Burton, W.B.: 1976, Astrophys.J. 208, 346.

Icke, V.: 1978, preprint.

Kaifu, J., Kato, T., Iguchi, T.: 1972, Nature Phys. Sc. 238, 105.

Kerr, F.J.: 1968, in Radio Astronomy and the Galactic System, IAU Symp. 31 , ed. H. van Woerden.

Kruit, P.C. van der: 1971, Astron. Astrophys. 13, 405.

Light, E.S., Danielson, R.E., Schwarzschi1d, M.: 1974, Astrophys.J. $194,257$.

Lynden-Be11, D.: 1969, Nature, 223, 690.

Matsuda, T., Ne1son, A.H.: 1977, Nature 266, 607.

Oort, J.H.: 1975, in Galactic Structure, Vol. V of Stars and Stellar

Systems, eds. Blaauw and Schmidt, University of Chicago Press, p.455.

Oort, J.H.: 1971, Nuclei of Galaxies, ed. D.J.K. O'Connel1, North Holland.

Oort, J.H.: 1977, Ann. Rev. Astron. Astrophys. 15, 295.

Oort, J.H., Plaut, L.: 1975, Astron. Astrophys. 41, 71 .

Ostriker, J.P., Peebles, P.J.E.: 1973, Astrophys.J. 186, 467.

Peters, W.L.: 1975, Astrophys.J. 195, 617.

Roberts, W.W.: 1971, Bul1. A.A.S. 3, 369.

Rougoor, G.W., Oort, J.H.: 1960, Proc. Nat1. Acad. Sci. U.S.A. 46, 1.

Sanders, R.H., Lowinger, T.: 1972, Astron.J. 77, 292.

Sanders, R.H., Prendergast, K.H.: 1974, Astrophys.J. 188, 439.

Scoville, N.Z.: 1972, Astrophys.J. 175, L127.

Simonson, S.C., Mader, G.L.: 1973, Astron. Astrophys. 27, 337.

\section{DISCUSSION}

Schmidt-Kaler: (1) The agreement between the density model from the infrared observations and the Sanders-Lowinger rotation curve (if these really agree) may be misleading. The $M / L$ rates need not be constant, because star formation is going on. (2) The rotation curve of the Galaxy inside $4 \mathrm{kpc}$ is certainly far from solid body. To explain just 
one feature, for just one time period, and from one observer's aspect is the wrong philosophy. Further, you consider viscous shear with slowly ingoing motions. I do not at all object to the existence of that shear but I believe a theory should first explain the whole situation of many features flowing out with high velocities. The philosophy of near-circular orbits for the gas and pure gravitation is probably just wrong in the innermost parts of the galaxy. (3) On the same grounds you obtain the great mass outflow rate, because you assume circular symmetry. Starting from a Riemann instability you find the outflow only at two diametrically opposite points or strips in the plane, and these points represent the onset of the spiral arms. A rate of $0.25 \mathrm{M}_{\Theta}$ /year is sufficient to maintain energy and momentum of the density wave in the main body of the Galaxy. That adds up to $\sim 10^{9} \mathrm{M}_{\odot}$ in those $5 \times 10^{9}$ years, corresponding to the age of the disk stars according to Demarque and McClure.

Sanders: (1) The Sanders-Lowinger rotation curve is the curve for a mass model derived from the near infrared observations. So, of course, they "agree". The statement was that this rotation curve is entirely consistent with the $21-\mathrm{cm}$ observations in the inner few degrees--and that is just a statement of fact. By playing with the assumptions that go into determination of the mass model from the near infrared observations, such as the mass-to-light ratio, one might be able to change the predicted rotation curve by $50 \mathrm{~km} \mathrm{~s}^{-1}$ or so, but not by much more. Your second point escapes me. I did not suggest that the rotation curve inside $4 \mathrm{kpc}$ is solid body; it is far from it. And it is possible to have apparent expansion due to flow on highly elliptical streamlines even in the presence of a low-velocity systematic inflow. (3) In your third point, I presume you are objecting to my arguments against an explosion model for the 3-kpc arm. It is true that the mass outflow rate can be lowered by proposing carefully aimed and highly directional ejection. But, I must admit, I do not understand your model.

van Woerden: There can be no objection to a model requiring two bars. Several external galaxies have two bars; e.g., NGC 1291 has a nuclear bar inside, and not aligned with, the major bar (see de Vaucouleurs 1975, Ap. J. Supp. 29, 193).

Sanders: That is a good point. In the case of our Galaxy, however, both bars would have to lie oriented in such a way that we would observe high-velocity outflow.

de Vaucouleurs: Instead of different ill-defined power laws for the space density distributions in the "nucleus", "bulge" and "halo", it is advisable to recognize that these are parts of a unique spheroidal component pervading the whole Galaxy from the innermost IR nucleus to the outermost globular clusters and obeying throughout the $R^{1 / 4}$ law in projection. P. Young has given (A.J. 1976) a convenient asymptotic expression for the space density distribution in a spheroid obeying the $\mathrm{R}^{1 / 4}$ law: 


$$
\rho(s) \simeq\left(\frac{\pi}{8 b j}\right)^{1 / 2} e^{-b j} / 2 j^{3},
$$

where $s=r / r_{c}=j^{4}$ and $b=7.669$. This is a very good approximation at all $\mathrm{s}>0.2(\sim 1 \mathrm{kpc}$ in our Galaxy). Monnet and Simien (A.\&A. 1977) have shown how a spheroid and exponential disk combination give good representations of the rotation curves of M31, M81.

Pişmiş: In the dynamical determination of mass one should be aware that the rotational velocity may deviate significantly from circular velocity The gravitational force, due to the mass interior to the point, is not necessarily balanced by the centrifugal acceleration at the point. The additional force is provided by the dispersion of the velocities. If this dispersion is not negligible, then the masses determined will be underestimated. These statements are based on the hydrodynamical equations of stellar synthesis.

Sanders: The 21-cm profiles in the inner few degrees are not compatible with a velocity dispersion in excess of $20 \mathrm{~km} \mathrm{~s}^{-1}$ or so. The pressuregradient force is not significant.

Yuan: From the observations of van der Kruit and Davies and Cohen, there are many expanding features in the central region. This seems to contradict the flows moving towards the nucleus, due to the loss of angular momentum, that you suggest in your theory. Could you comment on this?

Sanders: The inward flow of gas due to viscuous transfer of angular momentum is a very low-velocity flow $\left(2-3 \mathrm{~km} \mathrm{~s}^{-1}\right.$ at most), but it is systematic. The high expansion-velocity components which you refer to may not represent a systematic outflow. For example, it has been suggested that we might be observing an apparent expansion due to gas flow on highly elliptical streamlines in the field of a bar. In this case, it would be possible to observe an apparent expansion in the presence of a general low-velocity inflow. A gas "streamline" would look like this, in such a case:

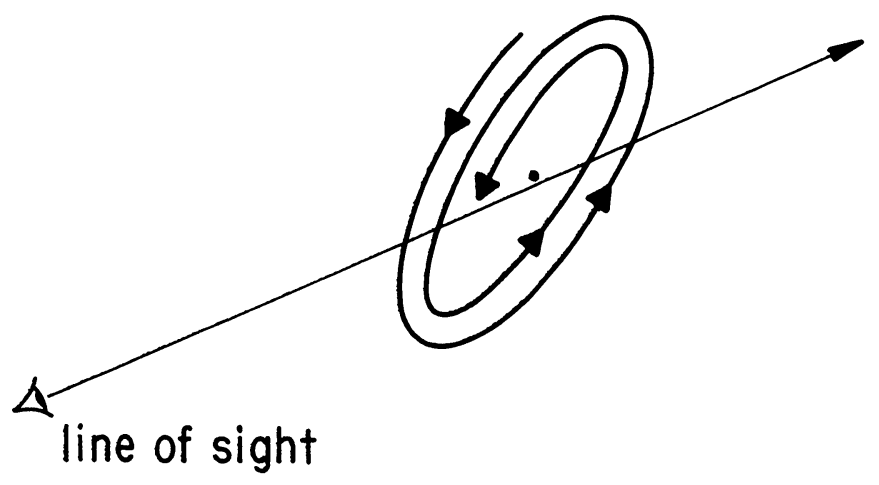

